\title{
ARTE PARA TODOS (Y TODAS): LA MEDIDA 40 EN EL PROGRAMA DE LA UNIDAD POPULAR
}

\section{ART FOR ALL MEN (AND ALL WOMEN): MEASURE 40 IN THE POPULAR UNITY PROGRAM}

\section{Carolina Espinoza Cartes*}

\section{RESUMEN}

El gobierno de la Unidad Popular otorga un papel activo y protagonista a la cultura como elemento transformador de la sociedad. Su presidente, Salvador Allende, cree fervientemente en que los derechos de los ciudadanos van más allá de la resolución de sus necesidades básicas y equipara en su programa de gobierno - las 40 medidas - derechos vanguardistas, tales como, el medio litro de leche diario para luchar contra la desnutrición infantil, con el acceso de los ciudadanos a una cultura popular $y$ descentralizada.

PALABRAS CLAVE: CHILE * CULTURA * ARTE * DEMOCRATIZACIÓN DE LA CULTURA * SALVADOR ALLENDE

\section{ABSTRACT}

The Popular Unity government grants an active and leading role to culture as a transforming element of society. Its president, Salvador Allende, fervently believes that the rights of citizens go beyond the resolution of their basic needs and equates in his government's program - the 40 measures - avant-garde rights, such as a pint of milk a day to fight child malnutrition, with citizens' access to a popular and decentralized culture.

KEYWORDS: CHILE * CULTURE * ART * DEMOCRATIZATION OF CULTURE * SALVADOR ALLENDE 


\section{INTRODUCCIÓN}

La cultura entendida en todas sus facetas jugó un papel clave en el gobierno de la Unidad Popular de Salvador Allende en Chile, que se extendió desde el 4 de noviembre de 1970 hasta el fatídico 11 de septiembre de 1973. El "hombre nuevo"1, concepto que será acuñado por Marx, luego por Gramsci y, posteriormente, por Ernesto Che Guevara (que resurge con fuerza con posterioridad a la Revolución Cubana) contempla no solo el acceso a los bienes materiales, sino a los que alimentan el espíritu, dando a las expresiones $y$ a las manifestaciones culturales un papel fundamental en su construcción y en la de una sociedad nueva (Marx, 1974; Gramsci, 1973; Guevara, 1977). Se trata de poner en el centro del análisis la cuestión de la cultura espiritual y el papel de la intelectualidad en el proceso revolucionario; así esta mirada inspira a los valores que generan la política cultural del gobierno de la Unidad Popular.

Otro factor que incide en la importancia de la cultura para este gobierno es el escenario mundial en que se forja. Chile vivía registrando cambios sociales y culturales con anterioridad a los acontecimientos ocurridos en Francia en mayo del 68. A diferencia de los cambios experimentados en Argentina o en Uruguay durante esta época, en Chile, el proceso de reforma universitaria había comenzado a inicios de los años 60 y continuado entre 1967-1970. Esto con la aplicación de medidas por parte de las autoridades, las cuales tendían a democratizar el funcionamiento de las universidades, a reorganizar las estructuras de enseñanza e investigación, renovar los métodos pedagógicos y abrir las instituciones al exterior. Su punto máximo se consolida al entrar en la Unidad Popular, teniendo como resultado una generación

1 Concepto acuñado por Marx para referirse al individuo que corresponde a la sociedad comunista, que permitirá el libre desarrollo pleno y armónico del hombre, un productor capacitado universalmente, conocedor de las bases científicas de la producción y con un pensamiento universal que le permita la plena satisfacción de las necesidades materiales y espirituales con un alto desarrollo ideopolítico, estético y moral. universitaria políticamente contestataria (Salazar y Pinto, 2002).

Tras el éxito de la reforma universitaria, la generación de 1968 en Chile, desencantada de la burocracia y la carrera política, prefiere acompañar a los movimientos sociales, ocupar los terrenos agrícolas incultos o las fábricas mal administradas. Se trata de una generación que quiere rebelarse contra las ataduras que conoce desde que nació. Este impulso por la democratización de la sociedad, sumado al halo de romanticismo que envuelve a todo proceso revolucionario latinoamericano en los años 60 , hace que esta generación se asuma como portadora de un cambio histórico (Boisard, como se citó en González y Sarriá, 2016) que los empujará a asumir con especial ímpetu los cambios culturales en este periodo.

Los jóvenes de la generación del 68 crecieron rodeados de gigantescas estructuras económicas, políticas e ideológicas: empresas multinacionales, estados burocráticos, confederaciones sindicales, bloques de países, imperialismos, sistemas mundiales de control monetario, planificaciones estratégicas, ejércitos con cohetes intercontinentales, grandes teorías ideologizadas, etc. Moles que se alineaban, además, en dos enormes bloques político militares que vivían en perpetua Guerra Fría (...) Por eso la generación del 68 se sintió compelida a ser, en ese escenario, un gigante moral. Un ejemplo de consecuencia. Y tuvo modelos a la mano: Ernesto Che Guevara o el pueblo vietnamita (Salazar y Pinto, 2002, p. 31).

Sí, el "mayo francés" influyó en el discurso de la Unidad Popular dirigido a las mujeres, que explica la enorme implicación de la mujer en todas las manifestaciones culturales. En el programa, se reivindicaba un mayor acceso a la formación universitaria, principal foco de cultivo del activismo social y político, $y$ en donde se fraguó una generación de chilenas politizadas que años después sufrirían el rigor represivo de la dictadura pinochetista (Maravall, 2012).

Todos estos factores, filosofía del "hombre nuevo", generación del 48, juventud e incorporación de la mujer, crearon un sustrato 
óptimo para que la política cultural del programa de la Unidad Popular se desarrollara a la perfección $y$, aunque no todas las expresiones tuvieron el mismo protagonismo, lo cierto es que con ellas se sentaron férreos sentimientos para el acceso a la educación y a la cultura, que resistieron firmemente al apagón cultural de la dictadura de Pinochet, que se prolongó por 17 años.

\section{UN CHILE BIEN DIFERENTE}

La nueva cultura expresada en la medida 40, además, debía abandonar el sistema patriarcal y proteccionista de la generación de políticas públicas desde la capital, y promover el surgimiento de medidas culturales acordes con las necesidades de cada provincia del país y beneficiar a través de estas medidas, a las clases más desposeídas.

Para tan ambiciosos objetivos, era claro construir una nueva cultura integradora que incorporara una visión desclasada, donde se abandonara el sentido elitista del concepto, privilegio de los grandes terratenientes y la clase media de la época.

El sistema de cultura popular estimulará la creación artística y literaria y multiplicará los canales de relación entre artistas o escritores con un público infinitamente más vasto que el actual (Medida 40 del Programa básico de Gobierno de la Unidad Popular, 1969, p. 28).

Esta nueva cultura se antepondría también a la sacralización "desde arriba", de las manifestaciones artísticas de la clase obrera, sin entrarse a examinar la calidad del mensaje emitido, cuyo contenido, por más que resulte expresión de una fábrica o una población, puede estar influido por la ideología dominante, como así ocurre, por caso, con ciertas expresiones espontáneas venidas de la plástica y de la representación teatral (Marín, 1972).

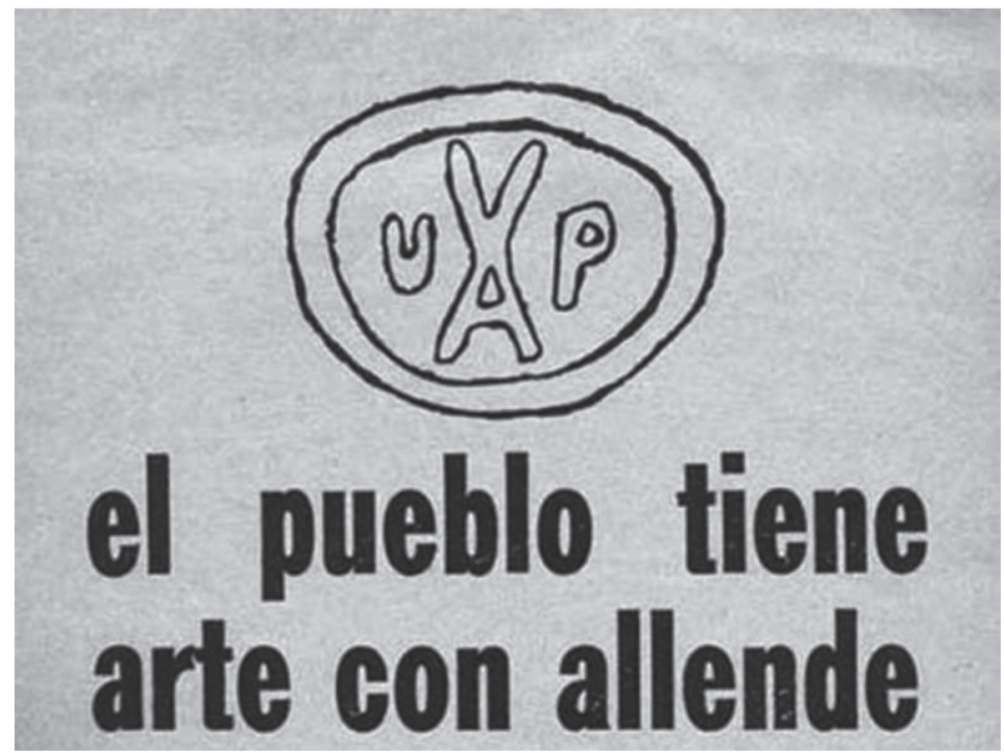

Fuente: Memoria chilena (http://www.memoriachilena.gob.cl/602/w3-channel.html). 


\section{EXPRESIONES CULTURALES}

Hablar de todas las manifestaciones culturales que tuvieron cabida en la política cultural del gobierno de la Unidad Popular no podría contenerse solo en un artículo. Esto porque el fenómeno se dio prácticamente en todas las expresiones que se declararon abiertamente a favor de los cambios estructurales y de la revolución social: la pintura y otras artes gráficas, el teatro, el cine y la literatura buscaron modos de expresión que dieran cuenta de su renovada voluntad crítica (Bowen, 21 de enero de 2008). Otros expertos, tales como César Albornoz (2005), prefieren hacer una selección, señalando que un importante espejo donde se reflejó el sentido y la identidad del proceso cultural en Chile bajo la Unidad Popular fue por sobre todo la música, la plástica y la industria editorial, las cuales marcaron una pauta $y$ se transformaron - años más tarde - en símbolo de aquella nueva sociedad truncada.

\section{ARTES ESCÉNICAS}

Las artes escénicas jugaron un papel fundamental en la política cultural del gobierno de la Unidad Popular. Las experiencias del teatro experimental, tanto de la Universidad de Chile como de la Universidad Católica, influyeron en las representaciones que comenzaron a exhibirse en los escenarios locales, motivadas por las corrientes rusas, francesas y españolas predecesoras.

Resulta evidente en la experiencia teatral, la influencia del proyecto renovador del teatro universitario español durante la segunda República, en especial, de La Barraca dirigido por Federico García Lorca en la Universidad de Madrid o El Búho, dirigido por Max Aub en la Universidad de Valencia. Tales experiencias habrían inspirado la creación de compañías de teatros universitarios en Chile. Esos grupos comenzaron en la época a realizar itinerancia por diferentes lugares de la geografía chilena y se inspiraron también en la experiencia del Teatro del Pueblo ${ }^{2}$ del asturiano Alejandro Casona, en

2 Teatro del pueblo y Teatro ambulante pertenecieron a la sección teatral de las Misiones su afán por embeber las problemáticas sociales del pueblo y reflejarlas en las obras.

La experiencia teatral en itinerancia y en especial en los pueblos más recónditos era muy interesante porque nosotros buscábamos al espectador, absorbiendo lo que ellos nos entregaban yendo al espectador, recogiendo la problemática que tenían y contándola teatralmente. Tuvimos experiencias de este tipo en Concepción, Valdivia, Temuco, lugares donde estaban los mapuches, en los reductos mapuche, y allí creo que fue más rica la experiencia (Pedro Villagra, entrevista al actor en el documental El Tren Popular de la Cultura, 2015).

En las artes escénicas se mezcló la representación de obras de teatro experimental, con otras del repertorio español tales como zarzuelas o la representación de clásicos de Miguel de Cervantes o Quevedo.

\section{MÚSICA}

Un gran número de artistas buscó llegar a través de sus producciones a estos nuevos sectores, al pueblo. Esto influyó, en el caso de la música, en la revalorización de las raíces folclóricas del país chileno, lo que determinó el surgimiento de La Nueva Canción Chilena, nacido en 1968, movimiento que en los años setenta alcanzó una importante difusión a través de la Discoteca del Canto Popular (DICAP), sello discográfico del Partido Comunista de Chile.

La mezcla entre música y política es lo que dará origen al neofolklore (Bowen, 21 de enero de 2008) con exponentes, tales como, Víctor Jara, Isabel y Ángel Parra, Patricio Manns o el grupo Quilapayún, quienes trabajaron en favor de la campaña presidencial de la izquierda en 1970, produciendo canciones destinadas

Pedagógicas creadas por Manuel Bartolomé Cossío, dentro del proyecto educativo del Gobierno de la Segunda República Española inspirado en la filosofía de la Institución Libre de Enseñanza. Estuvo dirigido por Alejandro Casona y formado por estudiantes universitarios (Gómez, 1997). 
específicamente a esa tarea. Pero el resultado fue más allá, al aprovechar toda la tradición poética que Chile había sabido guardar en sus raíces y mezclarlas con influencias de América Latina. El camino hacia la Nueva Canción chilena había sido pavimentado por folkloristas de la talla de Violeta Parra, Margot Loyola, Gabriela Pizarro o Héctor Pavez, junto con poetas como Pablo Neruda y Nicanor Parra, que incentivaron una labor musical a la que se sumó la influencia de compositores vecinos como Atahualpa Yupanqui o el cubano Carlos Puebla (Albornoz, 2005).

La experiencia fue plural y variopinta. Al llamado de gobierno acudieron artistas populares como el dúo Los Emigrantes, formado por Carlos Valladares y Enrique San Martín y dirigidos por Rolando Alarcón. También la música fue una instancia para jóvenes promesas como Fernando Nano Acevedo, quien supo canalizar el fervor de la Nueva Canción Chilena, a través de un acervo y una experiencia de participar entre 1966 y 1969, como cantautor en programas de radio $y$ festivales estudiantiles $y$ comunales, así como en la Peña "Chile Ríe y Canta" de René Largo Farías en 1968.

También el DICAP creó un espacio para la música clásica, que además de promover la Nueva Canción Chilena, quiso extender los clásicos musicales a los oídos de todo el pueblo. En esta última tarea, tuvo una especial participación el guitarrista Eulogio Dávalos, quien además de participar en la selección de clásicos, protagonizó conciertos clásicos populares junto al guitarrista argentino Miguel Ángel Cherubito.

Seguramente ese público no preparado, no había tenido una formación musical ni tan siquiera auditiva, pero el respeto que tenían por escuchar ese Bach, era algo que nunca dejaremos de pensar con una gran emoción. Después vendrá la intelectualización del arte, después vendrá el estudio académico, pero en ese momento el pueblo llano recibía con un respeto increíble, lo que nosotros ofrecíamos y para gran enseñanza nuestra, era lógicamente la esperanza de que la cultura, no pasa por grandes manifestaciones de enseñanzas, sino que es el sentimiento humano puro (Eulogio Dávalos, entrevista al guitarrista en el documental El Tren Popular de la Cultura, 2015).

También se desarrolló en este periodo, el folclore y la danza popular. El camino a esta última disciplina lo había trazado ya la artista Joan Turner, quien, junto a Alfonso Unanue, comenzó en 1964 a impartir talleres para aficionados.

En 1970, este mismo grupo de aficionados se constituyó con el nombre de Ballet Popular, el cual se convirtió en uno de los más importantes grupos de danza independiente de esos años por su autonomía económica y administrativa, así comopor su activa participación en la campaña presidencial de Salvador Allende, recorriendo plazas y poblaciones, con lo cual se llevaba la danza a todo el país, dando especial protagonismo a esta expresión popular y moderna del movimiento de danza contemporánea, la cual posteriormente sufrió una paralización con la dictadura.

\section{LITERATURA Y ARTES PLÁSTICAS}

El gobierno de la Unidad Popular adquirió la Editorial Zig-Zag para transformarla en la Editora Nacional Quimantú, que tuvo como propósito la edición a bajo coste de importantes títulos de la literatura nacional e internacional para poner destacadas obras literarias al alcance de todos. Allende dijo en su discurso inaugural en febrero de 1971:

Desde nuestro punto de vista, el paso que hemos dado significa el inicio de una nueva etapa en la difusión de la cultura en nuestro país. La nueva editorial del estado contribuirá eficazmente a la tarea de proveer a los estudiantes chilenos de sus textos de estudios, de promover la literatura nuestra $y$ de permitir que el libro sea un bien que esté al alcance de todos los chilenos(...) (Discurso inaugural presidente Allende, 13 de febrero de 1971, p. 5). 
Quimantú se transformó en el referente cultural del gobierno, a través de ediciones populares de revistas, documentos de trabajo e impresos necesarios para la política de democratización de la cultura. Famosas fueron también las colecciones de análisis sociológico de la idiosincrasia chilena Nosotros los chilenos dirigida por el poeta Alfonso Alcalde y los Cuadernos de Educación Popular, editados por Marta Harnecker y Gabriela Uribe. La editorial llegó a lanzar, en 1971, cerca de 50000 ejemplares de una obra, récord nunca más superado en el país.

En la poesía, durante la Unidad Popular no solo destacó la figura de los grandes, sino también de jóvenes poetas como Omar Lara, quien fue respaldado por el trabajo previo fundador en la revista Trilce en 1963. Lara, instalado en ese entonces en Valdivia, congregó a los poetas de cada región del sur de Chile, y fue natural ver en pueblos y regiones, lecturas dramatizadas de clásicos poéticos y talleres de poesía con los poetas de cada provincia que conformaban - en especial en el sur de Chile - un gran núcleo de producción poética.

Es así como el grupo Trilce, se había organizado en Valdivia bajo el amparo de la Universidad Austral y estaba integrado, además de su director Omar Lara, por Enrique Valdés, Juan Epple, Carlos Cortínez, Federico Schopf,
Luis Zaror y Eduardo Hunter. En Concepción, también había surgido por esos mismos años, el grupo Arúspice, apoyado por la Universidad de Concepción, integrado por Jaime Quezada, Silverio Muñoz, Floridor Pérez, Gonzalo Millán, José Luis Montero, Edgardo Jiménez, Ramón Riquelme y Raúl Barrientos. Ambos grupos con raíz local, participaron activamente en la dinamización de grupos.

En el ámbito plástico, participaron diversas escuelas y expresiones. Desde el muralismo que está en su momento cúspide a través de la figura de Julio Escámez — quien posteriormente se exilió en Costa Rica- las serigrafías de Mario Zapata en Tomé y Concepción, Martínez Santander, Aníbal Ortiz, Agustín Olavarría, Guillermo Tejeda o el acuarelismo de los pintores de la ciudad de Angol, encabezados por Miguel Ángel Roa.

Pero la expresión plástica que más se desarrolló tuvo que ver con otra vertiente más popular del muralismo: las brigadas muralistas, siendo la más reconocida, la brigada Ramona Parra, dependiente de las Juventudes Comunistas, que tuvo el respaldo de artistas plásticos como Roberto Matta. Tuvieron como primeros "lienzos" los bordes del río Mapocho, donde con colores resaltantes y figuras con una estética de la época, se contó la historia del movimiento obrero y del Partido Comunista chileno. 


\section{IMAGEN 2 \\ MURAL DE LA BRIGADA RAMONA PARRA EN EL CENTRO DE SANTIAGO DE CHILE DURANTE EL GOBIERNO DE LA UNIDAD POPULAR}

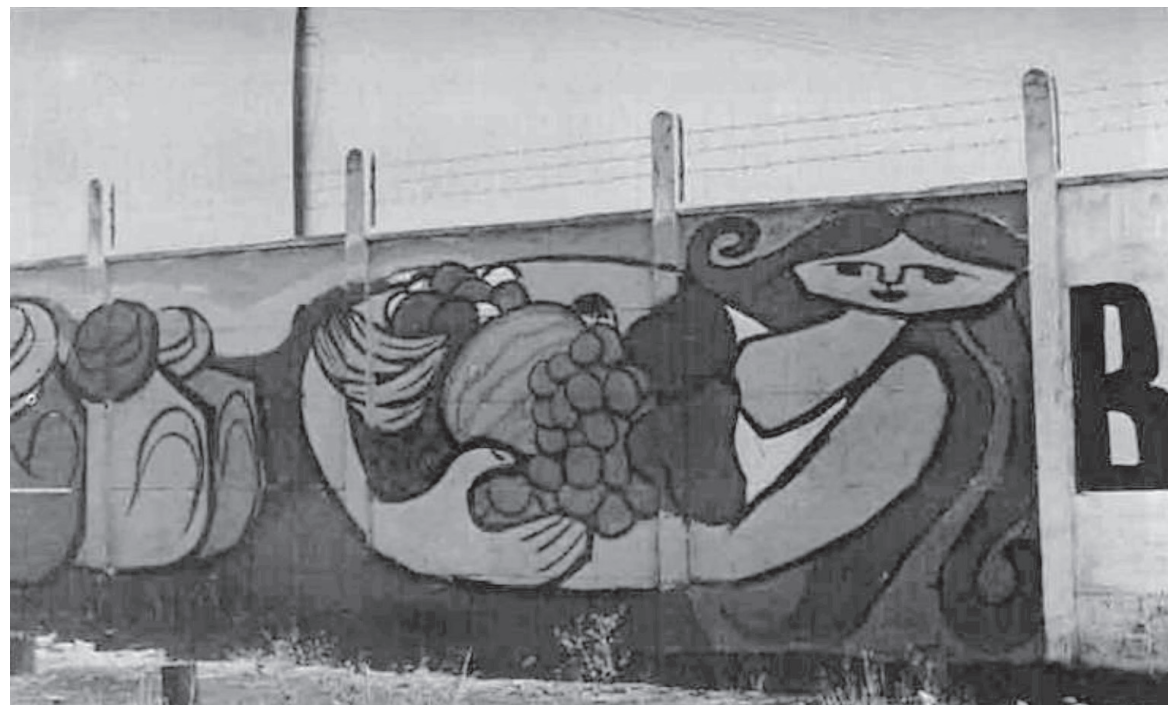

Fuente: Revista Araucaria, 1983.

El artista plástico Alejandro "Mono" González fue el director artístico de la Brigada Ramona Parra en 1971 y en ella encabezó proyectos murales que fueron más allá del compromiso político. Uno de ellos, el que fue emplazado en la comuna de La Granja titulado "El primer gol del pueblo chileno" que fue borrado con 16 capas de pintura durante la dictadura y restaurado en 2008 . En un documental homónimo que explica el proceso de restauración (2008), González explica:

Con la campaña de Allende, el movimiento brigadista se extiende a nivel nacional y la tarea era conquistar todos los muros para el pueblo. Los periodistas extranjeros que vinieron a cubrir el triunfo de Allende en septiembre de 1970, se fijaron en los murales $y$ ahí nos empezaron a tomar en serio este nuevo concepto de hombre nuevo que nosotros reflejábamos en los muros. Hay un despertar, una explosión creativa que tiene su origen en el avance social del pueblo (Alejandro González, entrevista en el documental El mono y el primer gol del pueblo chileno, 2008).

\section{EL TREN POPULAR DE LA CULTURA}

La creación de los Institutos Provinciales de la Cultura fue el objetivo final de la promoción de la medida 40 expresada en la realización del Tren Popular de la Cultura. La idea, encomendada directamente por el presidente Allende al departamento de Cultura, consistía en fletar un tren rumbo al sur para lograr que en cada provincia, de acuerdo a su realidad e identificación cultural, se creara una nueva institucionalidad cultural, donde fuera posible desarrollar el valor de los profesionales sin extraerles de su entorno cultural, con el fin de agilizar la devolución y que esta repercutiera en su desarrollo. Estos a su vez, conformarían el Instituto Nacional del Arte y la Cultura, representante máximo de la cultura en el gobierno. Si bien, el objetivo final no se llegó a concretar, sí se forjó la necesidad de no depender de las medidas culturales centralizadas. 


\section{IMAGEN 3 \\ PARTIDA DESDE LA ESTACIÓN CENTRAL DE SANTIAGO DE CHILE \\ DEL TREN POPULAR DE LA CULTURA \\ FEBRERO, 1971}

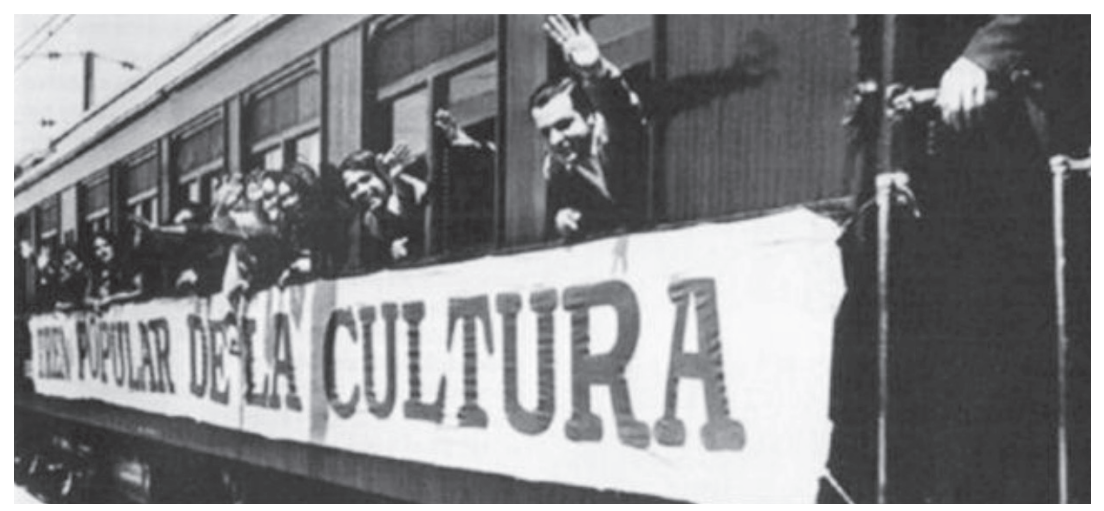

Fuente: Archivo Eulogio Dávalos.

Puede decirse que el Tren Popular de la Cultura fue la primera representación popular fuera de la capital del país, de inclusión de las masas a la actividad artística. Estuvo inspirada en el espíritu de las Misiones Pedagógicas de la segunda República española, a su vez, emuladas por varias experiencias similares en Colombia, Cuba, México y Uruguay, así como en los trenes culturales agitrop de la Revolución Rusa.

En este participaron sesenta artistas de las más diversas disciplinas artísticas durante cuarenta días por el sur de Chile, desde Puerto Montt hasta Santiago. La experiencia se realizó en febrero de 1971 y no se repitió al año siguiente, ante el estado de tensión política en que se sumió el gobierno popular.

\section{CONCLUSIONES}

Las políticas culturales del gobierno de la Unidad Popular encabezado por el gobierno de Salvador Allende, tuvieron por objetivo la incorporación de masas a la actividad artístico cultural, el goce y el disfrute de la cultura, unos beneficios que hasta ahora y en especial en Latinoamérica, estaban reservados para las clases más acomodadas.
La medida 40 del programa de gobierno, trataba de potenciar el poder transformador de la cultura en la sociedad, proporcionándole la misma importancia que medidas tan vanguardistas para la época, como el derecho del medio litro de leche a cada niño para combatir la desnutrición o los cambios en la educación, que resultan para la época innovadores e inclusivos.

Para conseguir que las personas de lugares más apartados disfrutaran por igual de la cultura y el arte, se tuvo en cuenta la ruptura de un eje centralizador como el que usaron hasta ese momento los gobiernos de la época. La medida 40 impulsó la irrupción del factor no paternalista $y$ descentralizado de la cultura, promoviendo que artistas de provincias tuvieran las mismas posibilidades de desarrollar una carrera artística sin emigrar a la capital. Por esta razón, la medida 40 dejó expresamente señalada la creación del Instituto Nacional del Arte y la Cultura a través de la instauración de escuelas de formación artística en todas las provincias de Chile.

Este devenir de fuerzas se aprovechó de comprobar in situ y de forma masiva, experiencias vanguardistas de la época como la nueva 
mirada al muralismo, la música de raíz folclórica, la danza o el teatro experimental.

Las circunstancias políticas de los siguientes años de la Unidad Popular no propiciaron un desarrollo más allá del activismo partidista de estas expresiones, que quedaron durante los primeros años circunscritas a experiencias puntuales $y$ vinculadas con objetivos electorales. Sin embargo, por su entusiasmo y efecto en la población, la experiencia sembró una "sed cultural" que fue aplacada a través de una potenciación de la cultura en provincias, con destacados avances en el ámbito de la literatura, las artes plásticas y el teatro.

Tras el golpe de Estado de 1973, muchos de los artistas y funcionarios que participaron en estas experiencias fueron perseguidos, detenidos, torturados y empujados al exilio. Desde cualquiera de esas circunstancias, siguieron apegados a la actividad cultural, cumpliendo un compromiso tácito $y$ de por vida con el programa cultural de Allende y la Unidad Popular.

Tanto caló en sus vidas este compromiso, que los sesenta participantes del Tren Popular de la Cultura siguieron desarrollando hasta los últimos días de su vida la actividad cultural, y tratando de involucrar a sus entornos más cercanos y masivos, para cumplir el mandato del programa de gobierno popular: conseguir que el arte, sea para todos ( $y$ todas).

\section{REFERENCIAS}

Albornoz, C. (2005), La cultura en la Unidad Popular: porque esta vez no se trara de cambiar a un presidente. En Pinto, J. (Ed.), Cuando hicimos historia: la experiencia de la Unidad Popular (pp. 147176). LOM.

González, C. y Sarrià, A. (Eds.) (2016). Militancias radicales. Narrar los sesenta y setenta desde el siglo XXI. Postmetropolis Editorial-Prohistoria Ediciones.

Bowen, M. (21 de enero de 2008). El proyecto sociocultural de la izquierda chilena durante la
Unidad Popular. Crítica, verdad e inmunología política. Nuevo Mundo Mundos Nuevos, Debates. DoI: https://doi. org/10.4000/nuevomundo.13732

Dávalos, E. (2015). Entrevista en documental Tren Popular de la Cultura (España, 60').

Discurso inaugural presidente Allende. (13 de febrero de 1971). Diario La Nación, p. 5.

Gómez García, M. (1997). Diccionario del teatro. Ediciones Akal.

González, A. (2008). Entrevista en documental El mono del primer gol del pueblo chileno (Chile, 20'). [Archivo de Vídeo]. Youtube. https://www.youtube.com/ watch? $v=Y C 2 A 4 Y \operatorname{trKVU}$

Gramsci, A. (1973). Antología. Editora Ciencias Sociales, La Habana.

Guevara, E. (1977). Escritos y Discursos (T 8, p. 79, 13). Editora de Ciencias Sociales, La Habana.

Marín, G. (28 de julio-3 de agosto de 1972). "Elitismo o Populismo: falsa alternativa". Chile, hoy, 7, 19.

Maravall, J. (2012). Las mujeres en la izquierda chilena durante la Unidad Popular y la Dictadura Militar [Tesis doctoral, UAM, Madrid].

Marx, C. y Engels, F. (1974). La ideología alemana. O.E. (Tres tomos). Grijalbo.

Medida 40 del Programa básico de Gobierno de la Unidad Popular. (1969). Candidatura presidencial de Salvador Allende, p. 28.

Revista Araucaria de Chile. (1983). https:// www.archivochile.com/Cultura_Arte_ Educacion/araucaria/araucaria23.pdf. Ediciones Michay.

Salazar, G. y Pinto, J. (2002). Historia contemporánea de Chile V. LOM Ediciones.

Villagra, P. (2015). Entrevista en el documental El Tren Popular de la Cultura (España, 60).

Fecha de ingreso: 28/08/20 Fecha de aprobación: 24/11/20 
